\title{
Impact of an Exhaust Throat on Semi-Idealized Rotating Detonation Engine Performance
}

\author{
Daniel E. Paxson \\ NASA Glenn Research Center \\ Cleveland, Ohio
}

\author{
SciTech 2016 \\ 54 ${ }^{\text {th }}$ AIAA Aerospace Sciences Meeting \\ San Diego, CA \\ January 4-8, 2016
}




\section{Outline}

- Background

- Problem Statement

- Problem Analysis

- Accommodation Strategy

- Results

- Concluding Remarks 


\section{Background}

Rotating Detonation Engines (RDE's) represent an Intriguing Approach to Detonative Pressure Gain Combustion (PGC)

PGC: A periodic process, in a fixed volume, whereby gas expansion by heat release is constrained, causing a rise in stagnation pressure and allowing work extraction by expansion to the initial pressure.

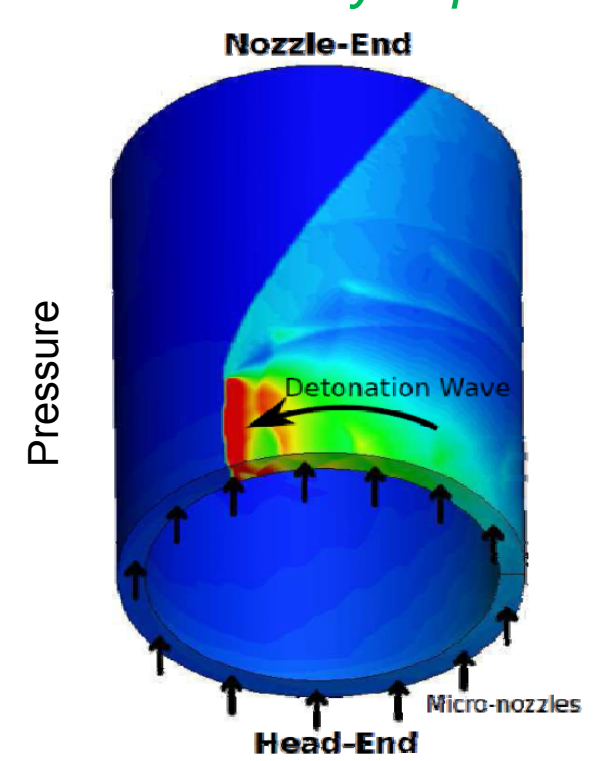

Source: Schwer, AIAA 2011-581

-1000+ Hz. cycle frequency

- No 'spark' required

- No lossy DDT devices

- Compact
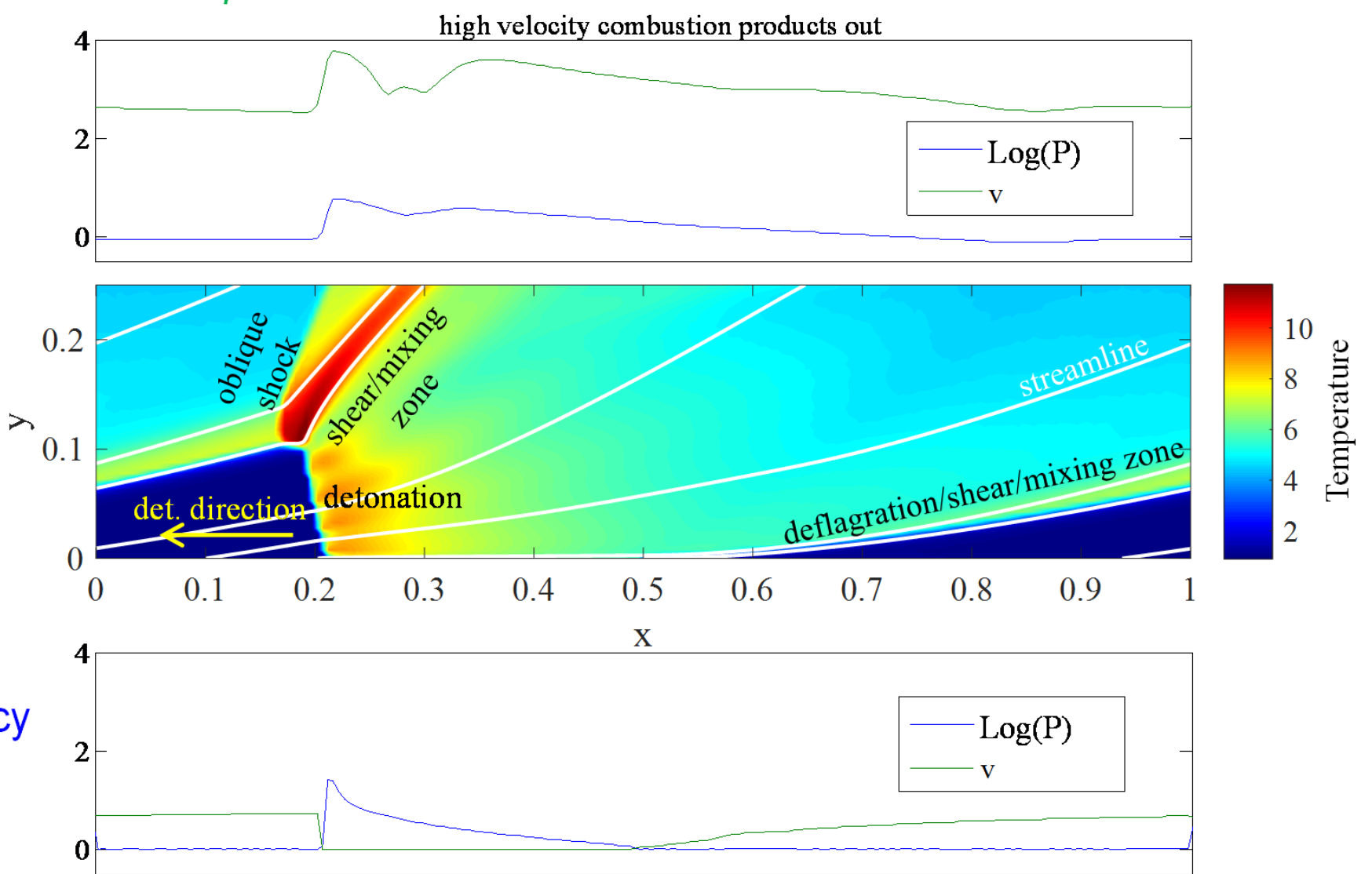

low velocity detonable mixture in 


\section{Problem Statement}

Consider a Semi-Ideal, Ram-Based, Stoichiometric Hydrogen Fueled

RDE at 37,000 ft., Flying at Mach 1.37

(Note-Flight conditions are illustrative only)

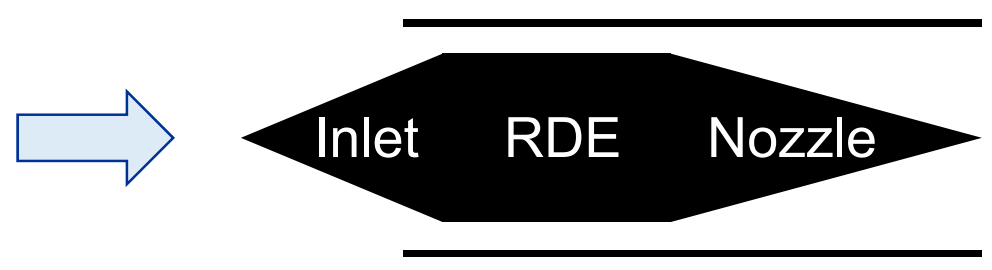

- Semi-Ideal Means

- Mil. Spec. engine inlet

- Combustor (RDE) inlet is lossless

- Combustor inlet has no reverse flow (i.e. perfect valve)

- Engine exit nozzle is lossless (i.e. perfectly expanded)

- Adiabatic

- Inviscid

- Premixed

- Retains fundamental entropy sources associated with RDE's 


\section{Problem Statement}
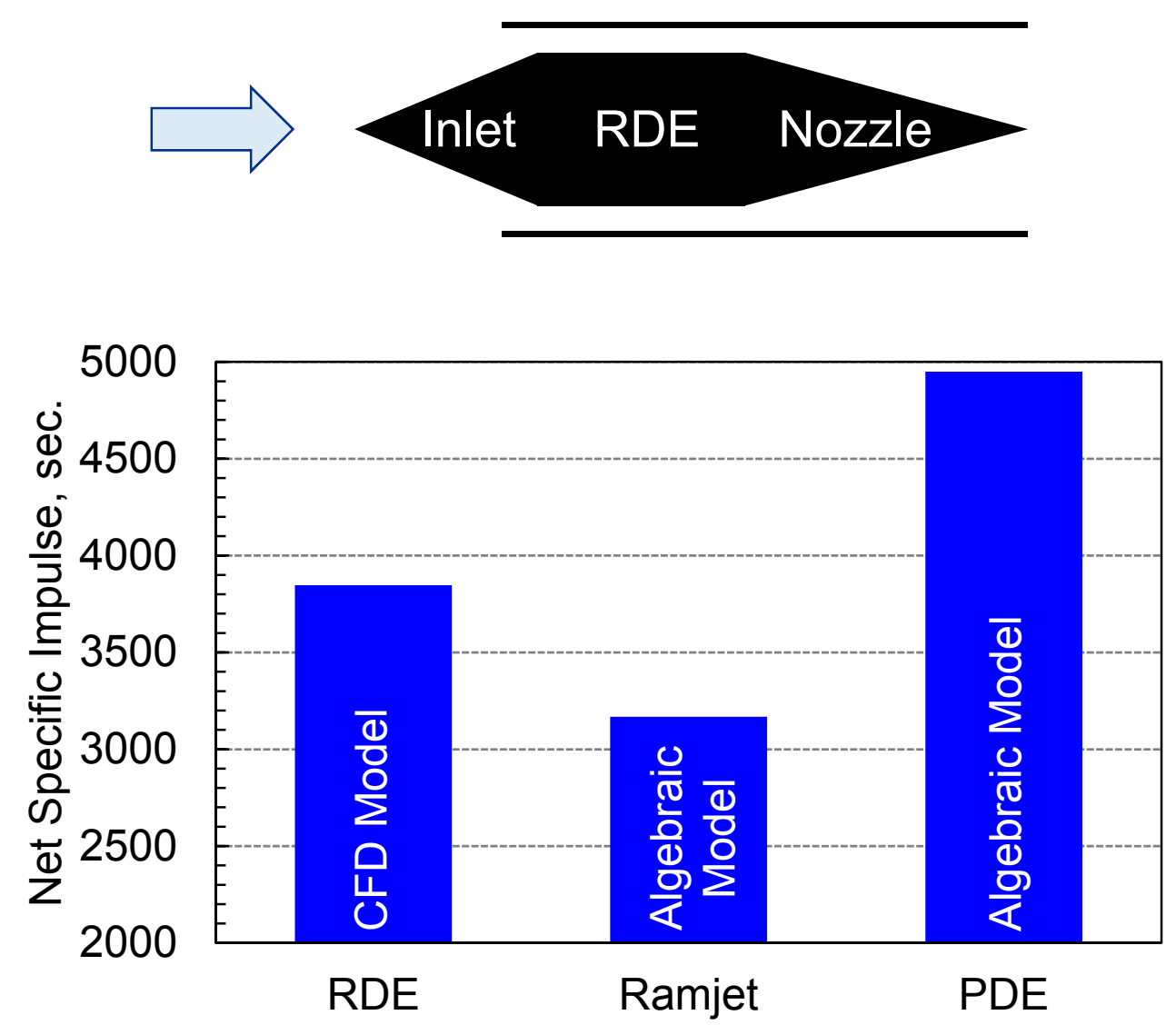

- RDE $21 \%$ above RJ

- RDE $22 \%$ below PDE ? 


\section{Problem Statement}

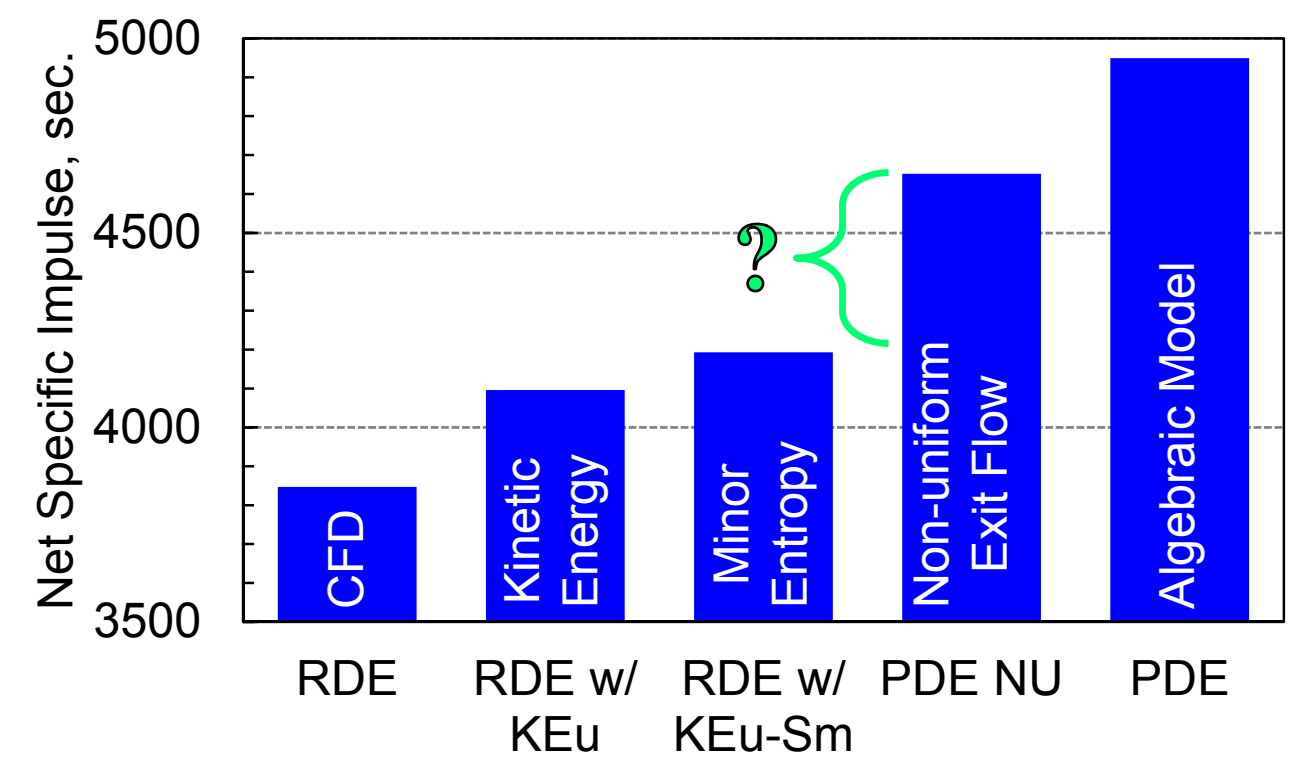

Contours of Entropy Relative to Algebraic PDE highest entropy flow

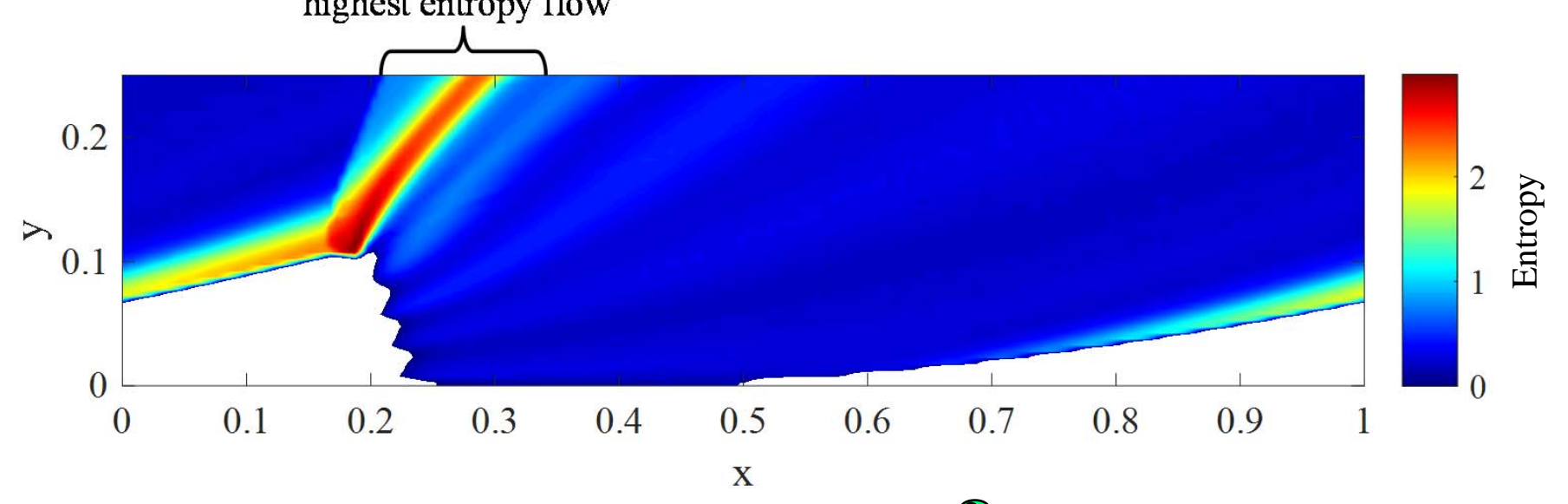

- $7-10 \%$ Disparity?

Where's All This Blue Coming From and What Can Be Done About it? 


\section{Problem Analysis \\ Primary Analysis Tool}

Quasi-2-Dimensional Euler Solver With Sources

- Source Terms Model:

- Chemical Reaction

- Friction (not used here)

- Heat Transfer (not used here)

- 2 Species Reaction (reactant or product)

- Simplified Finite Rate Reaction

- High Resolution Numerical Scheme

- Coarse Numerical Grid (<10,000 cells)

- Adopts Detonation Frame of Reference

- Time derivatives ultimately vanish and solution is steady

- Robust Boundary Conditions

- Sub or supersonic exhaust flow

- Optional isentropic exhaust throat

- Forward or reverse inlet flow with choking possible (not used here)

- Physics based inlet loss model from typical restriction (mostly not used here)

- Runs on a laptop

- Approximately $20 \mathrm{sec}$. per wave revolution

- Validated

- Compares well with other semi-idealized numerical results

- Compares well with experimental results 


\section{Problem Analysis}

\section{Effects of Fill Mach Number On 1D PDE}

-Algebraic 1D PDE Results Assumed Low Fill Mach Number

- As Fill Mach Increases Post Detonation Entropy Increases and Specific Impulse Decreases

- As Fill Mach Increases Predetonation Pressure Drops

- Detonation Does Not Recover Pressure

- So Post-Detonation CJ Pressure Drops

- Less Availability for Thrust Production
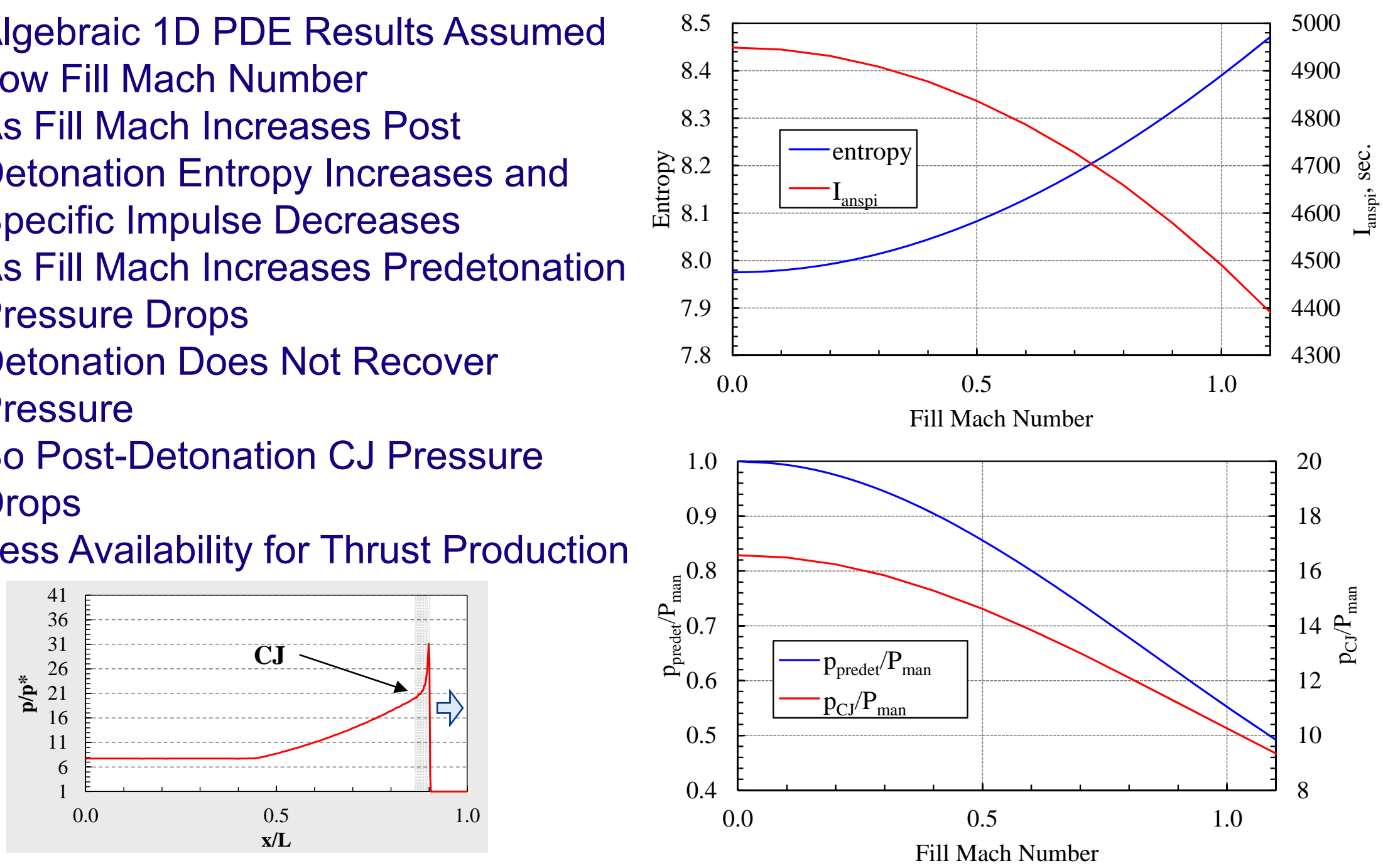

Analytical Results for 1D PDE's Say Fill Mach is the Culprit 


\section{Problem Analysis Effects of Fill Mach Number On RDE}

-Fill Mach Number Tricky to Define

- Using axial Mach number just prior to detonation

-Axial Mach Number Is High

-Post-Detonation Entropy Is High

- Fill Mach and Entropy Follow Same Relationship as 1D PDE
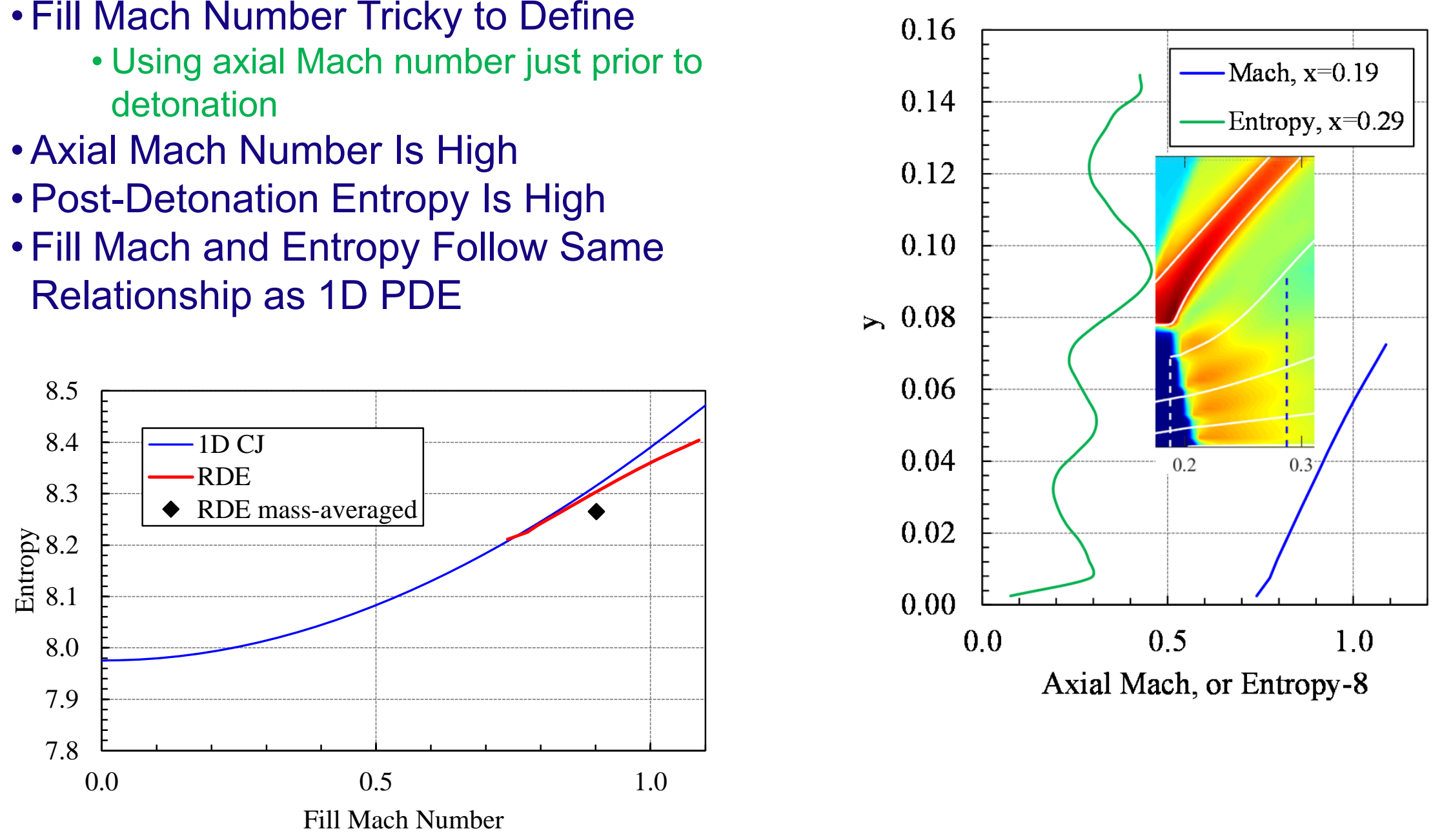

CFD Results for RDE's Suggest Fill Mach is Indeed the Culprit 


\section{Accommodation Strategy Add an Exit Throat}

- Rate of Exhaust Affects Rate of Fill

- Well established from PDE efforts

- Lower Rate of Fill Yields Higher Pre-detonation Pressure, Higher Post-Detonation Pressure, Lower Entropy, Higher Specific Impulse
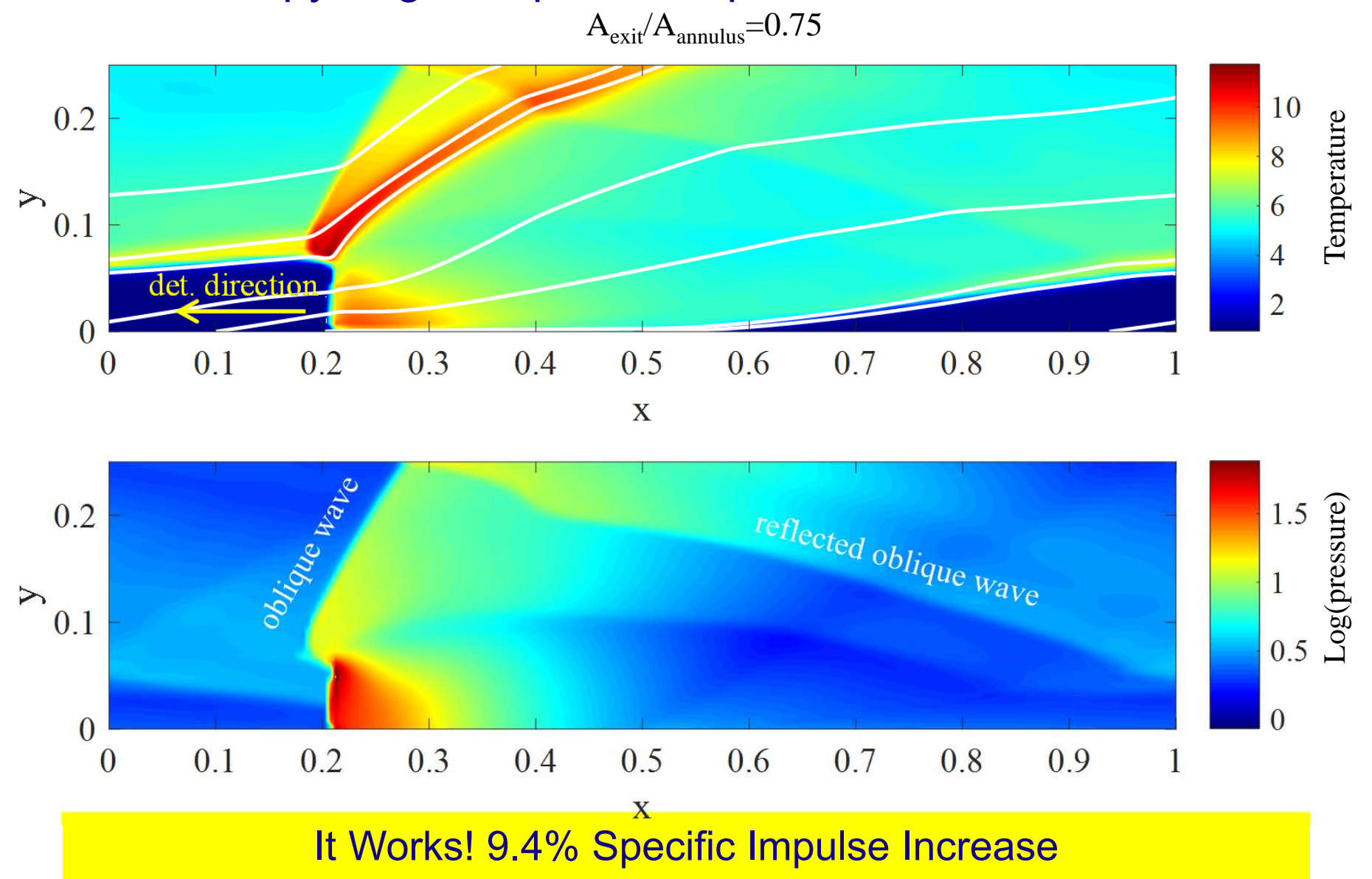


\section{Accommodation Strategy}

More Restriction!

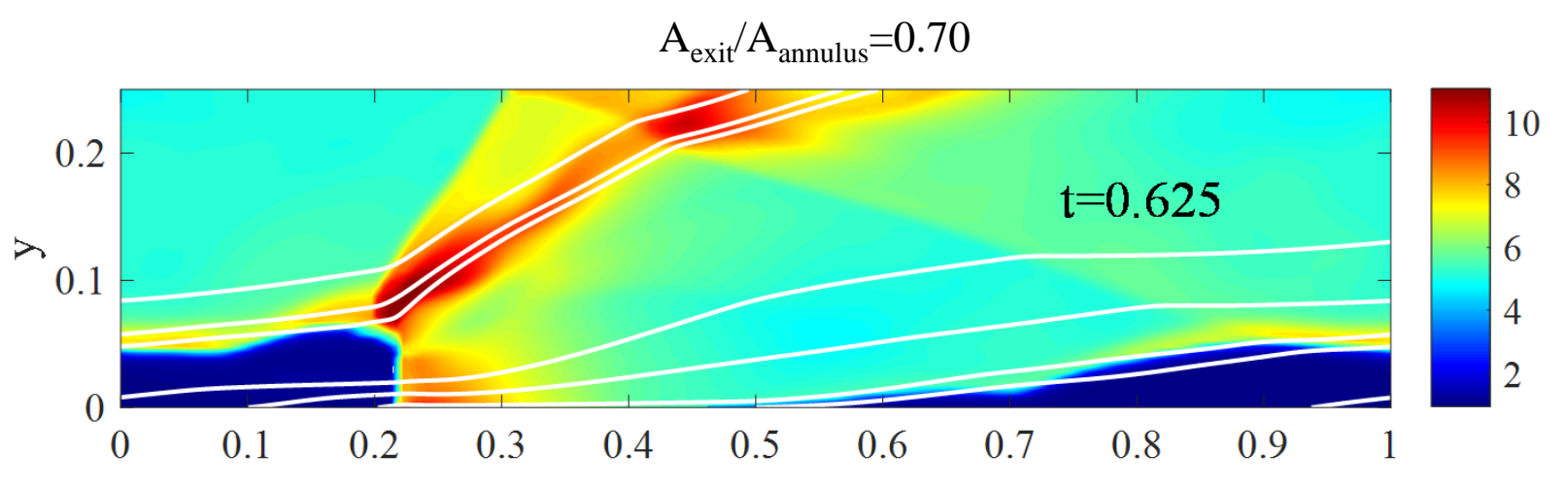

- Throat Sends Strong Waves Upstream

-Waves Affect Inflow

- Inflow Changes Affect Detonation Structure

- Detonation Changes Generate Additional Spurious Waves

-Waves Get Reflected

- Cascade Established
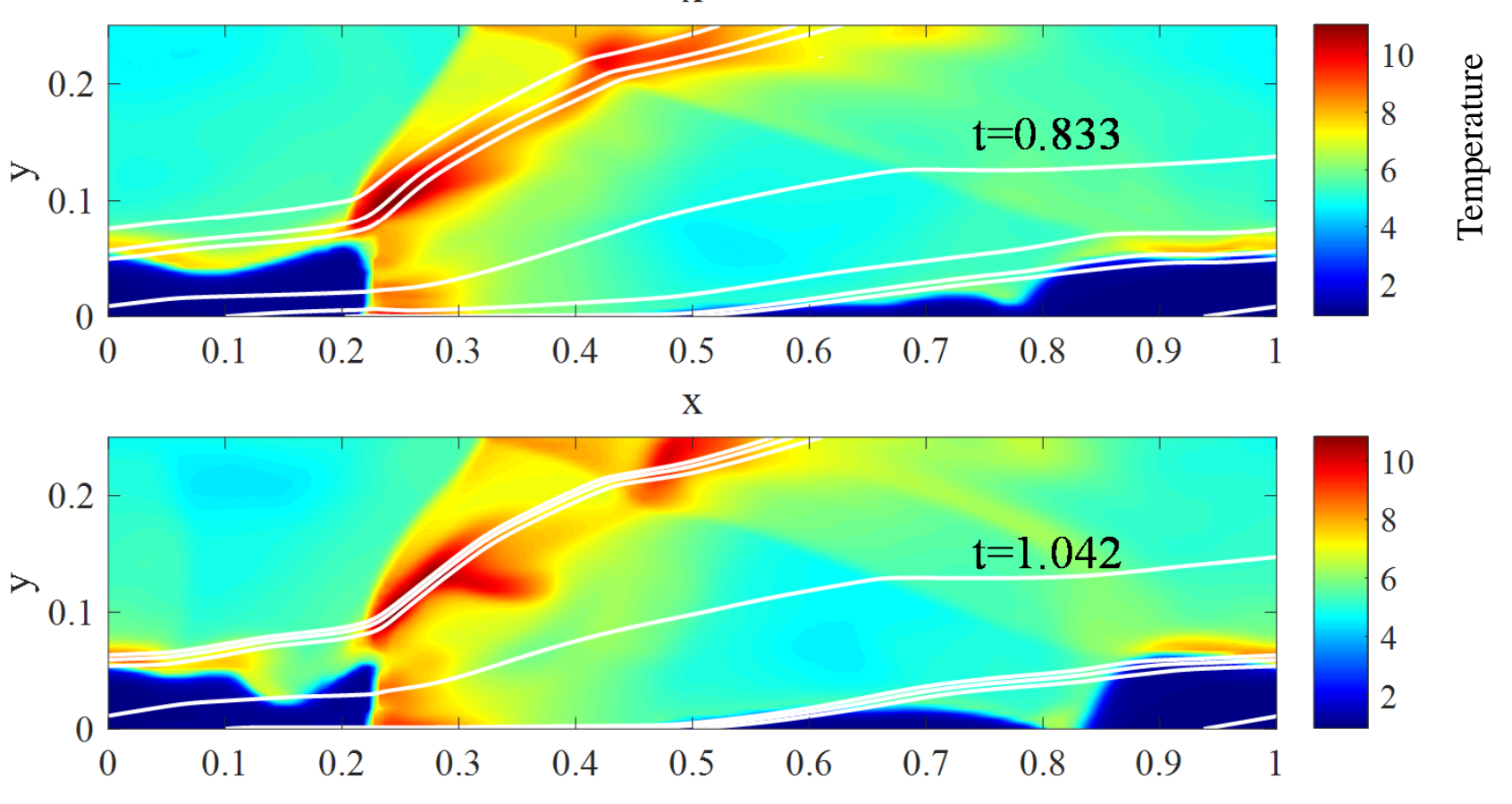

Unstable Behavior Results 


\section{Accommodation Strategy} Inlet Restriction With Loss

- Inlet Restriction Creates Total Pressure Loss...

-But Damps Unstable Behavior Allowing Smaller Exit Restrictions...

- Ultimately Yielding Net Gain
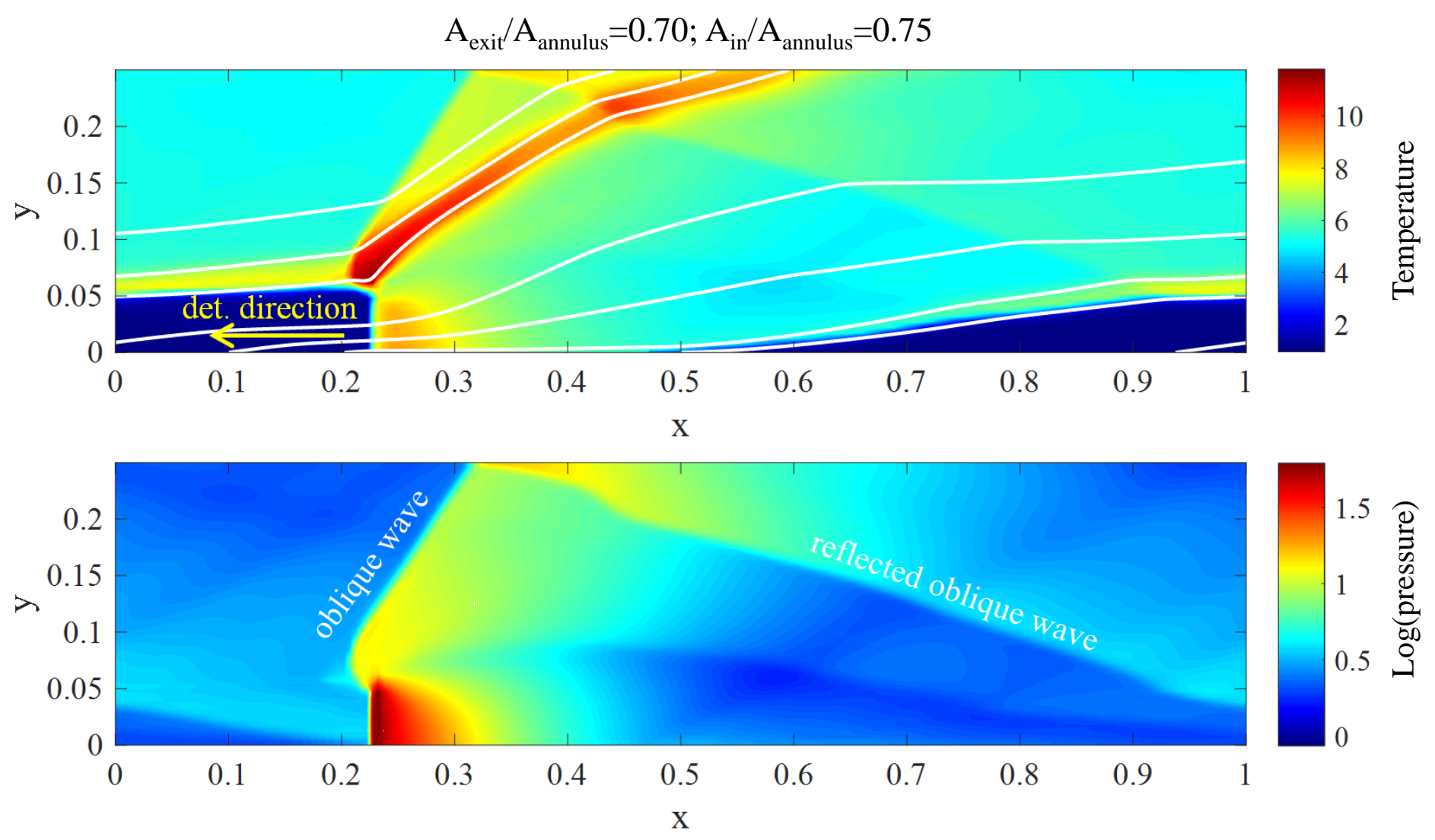

$10.3 \%$ Specific Impulse Increase 


\section{Concluding Remarks}

- For an idealized, basic RDE configuration, the fill Mach number can be quite high under representative boundary conditions

- Through the same basic mechanism as the PDE, the high fill Mach limits performance as measured by net specific impulse

- The fill Mach can be reduced by adding a throat to the exit, thereby gaining as much as $9 \%$ net specific impulse

- Too much exit restriction yields unstable operation

- Adding a 'lossy' inlet restriction adds stability and allows for a $10 \%$ specific impulse improvement

- Experimental validation (or refutation) is justified 
